\title{
Prevalence of metabolic syndrome in type 2 diabetic patients: A comparative study using WHO, NCEP ATP III, IDF and Harmonized definitions
}

\author{
Mun Chieng $\operatorname{Tan}^{1^{*}}$, Ooi Chuan $\mathrm{Ng}^{2 \#}$, Teck Wee Wong ${ }^{3}$, Anthony Joseph ${ }^{4}$, \\ Yoke Mun Chan ${ }^{5}$, Abdul Rahman Hejar ${ }^{6}$ \\ ${ }^{1}$ Department of Nutrition and Dietetics, Faculty of Medicine and Health Sciences, Universiti Putra Malaysia, Selangor, Malaysia; \\ *Corresponding Author: mun chieng tan@yahoo.com \\ ${ }^{2}$ Department of Medicine, Faculty of Medicine and Health Sciences, Universiti Putra Malaysia, Selangor, Malaysia; \\ \#Main Corresponding Author: chuan@medic.upm.edu.my, drocng@yahoo.com \\ ${ }^{3}$ Heart and Lung Centre, iHEAL Medical Centre Kuala Lumpur, Federal Territory of Kuala Lumpur, Kuala Lumpur, Malaysia \\ ${ }^{4}$ Department of Medicine, Faculty of Medicine and Health Sciences, Universiti Putra Malaysia, Selangor, Malaysia \\ ${ }^{5}$ Institute of Gerontology, Universiti Putra Malaysia, Selangor, Malaysia \\ ${ }^{6}$ Department of Community Health, Faculty of Medicine and Health Sciences, Universiti Putra Malaysia, Selangor, Malaysia
}

Received 24 July 2013; revised 24 August 2013; accepted 10 September 2013

Copyright (C) 2013 Mun Chieng Tan et al. This is an open access article distributed under the Creative Commons Attribution License, which permits unrestricted use, distribution, and reproduction in any medium, provided the original work is properly cited.

\section{ABSTRACT}

To determine the prevalence of metabolic syndrome (MetS) in Malaysian type 2 diabetic patients using WHO, NCEP ATP III, IDF and the new Harmonized definitions, and the concordance between these definitions. This study involved 313 patients diagnosed with type 2 diabetes mellitus (T2DM) at two Malaysian tertiary hospitals. Socio-demographic data were assessed using a pre-tested interviewer-administered structured questionnaire. Anthropometric measurements were carried out according to standard protocols. Clinical and laboratory characteristics were examined. Kappa $(\kappa)$ statistics were used for the agreement between the four MetS definitions. The overall prevalence rates of MetS $(95 \% \mathrm{Cl})$ were $95.8 \%$ (93.6 - 98.1), 96.1\% (94.0 98.3), 84.8\% (80.8 - 88.9) and 97.7\% (96.1-99.4) according to the WHO, NCEP ATP III, IDF and the Harmonized definitions, respectively. The Kappa statistics demonstrated a slight to substantial agreement between the definitions $(\kappa=0.179$ $0.875, p<0.001$ ), where the WHO criteria revealed the highest concordance with the NCEP ATP III definition $(\kappa=0.875, p<0.001)$. The WHO against NCEP ATP III criteria evinced the highest sensitivity (99.66\%) whereas Harmonized criteria against all the other three definitions showed the highest specificity (100\%) in identifying MetS. In conclusion, the new Harmonized criteria established the highest prevalence of MetS among the four definitions applied. There was a very good concordance between the WHO and NCEP ATP III criteria. The extremely high prevalence of MetS observed in type 2 diabetic patients indicates an impending pandemic of CVD risk in Malaysia. Aggressive treatment of MetS components is required to reduce cardiovascular risk in T2DM.

Keywords: Metabolic Syndrome; Type 2 Diabetes Mellitus (T2DM); World Health Organization (WHO); Third Report of the National Cholesterol Education Expert Panel on Detection; Evaluation; Treatment of High Blood Cholesterol in Adults (NCEP ATP III); International Diabetes Federation (IDF); Harmonized Definition

\section{INTRODUCTION}

Metabolic syndrome (MetS) is a cluster of metabolic abnormalities that often co-exist and would lead to a marked increase in the risk of cardiovascular disease (CVD) and type 2 diabetes mellitus (T2DM) including obesity, hyperglycemia, dyslipidemia and hypertension [1-7]. The essence of the MetS lies in the clustering of these risk factors, whose presence has extensively been reported to point to an almost five-fold elevation in CVD risk [6,8-11]. Metabolic syndrome is common in individuals with diabetes mellitus (DM) and significantly more common in patients with T2DM than in those with type 1 diabetes mellitus (T1DM) [12-14]. The total CVD 
risk attributable to the syndrome has been observed to exceed the sum of the risk from each of the separate components $[8,15]$. Hence, it becomes a great burden on public health and clinical practice [16].

Metabolic syndrome consists of a multi-factorial set of indicators [2-4,7,16-19]. The World Health Organization (WHO) definition [5] was the first to tie together the key components of MetS: insulin resistance, obesity, dyslipidemia and hypertension, where the presence of insulin resistance is mandatory. With that said, this definition also allows patients with T2DM to be diagnosed with MetS if they meet the other criteria.

In 2001, the National Cholesterol Education Program Adult Treatment Panel III (NCEP ATP III) devised a definition for MetS [3], which was then updated in 2005 by the American Heart Association/National Heart, Lung, and Blood Institute (AHA/NHLBI) [20]. The NCEP ATP III definition did not require demonstration of insulin resistance per se and can be used in people with DM. Moreover, no single factor is essential, instead, NCEP ATP III requires the presence of three out of any five factors to establish the MetS diagnosis. The NCEP ATP III provides a definition of the MetS that is pragmatic, applicable to routine clinical practice and uses variables that are easily measurable.

In 2005, the International Diabetes Federation (IDF) published new criteria for MetS [17]. Although it includes the same general criteria as the other definitions; it requires obesity, but not necessarily insulin resistance, to be present. The obesity requirement is met by population-specific cut-points. This accounts for the fact that different populations, ethnicities and nationalities have different distributions of norms for body weight and waist circumference (WC). It also recognizes that the relationship between these values and the risk for CVD differs in different populations. For example, Asian populations have an increased risk for CVD at smaller waist circumferences that would not be considered to meet the criteria in a Western population [21].

Recently, the IDF, AHA/NHLBI, World Heart Federation, International Atherosclerosis Society, and International Association for the Study of Obesity jointly proposed a Harmonized definition for MetS [7]. By this definition, the five risk factors were identical to the IDF criteria but did not mandate abdominal obesity as a compulsory risk factor. This means any three abnormal findings out of the five would qualify a person for the MetS. Thus, the definition is not built in any preconceived notion of the underlying cause of MetS, whether it is insulin resistance/DM or obesity.

There is limited data on the prevalence of MetS among Malaysian type 2 diabetic patients and the agreement between various MetS definitions in this population. In this study, the four most popular definitions proposed by different world medical organizations were applied to define and compare the complexity of different MetS among the type 2 diabetic patients in our tertiary hospitals. We estimated the overall MetS prevalence rates among these patients, followed by quantification and subsequent comparison of the degree of agreement between the available MetS definitions.

\section{METHODS}

\subsection{Patients and Study Location}

In this cross-sectional study, we studied 313 type 2 diabetic patients from two tertiary referral hospitals in Klang Valley, Malaysia-Kuala Lumpur Hospital and Serdang Hospital. A systematic random sampling method was applied to select patients based on the inclusion and exclusion criteria. The study protocol conforms to the principles of the Malaysian Guideline for Good Clinical Practice [22] which were consistent with the Ethical Guidelines of the Declaration of Helsinki (World Medical Association Declaration of Helsinki) as reflected in priori approvals by the Committees for Medical Research Ethics of the Faculty of Medicine and Health Sciences, Universiti Putra Malaysia, and Ministry of Health Malaysia on the understanding that patients' data were coded and anonymity guaranteed. Additionally, all patients were aware of the nature of the study and gave informed consent prior to commencement of the interview.

Comprehensive information on patients was collected using structured questionnaire, physical examination and laboratory investigations according to standardised protocols. Patients were personally interviewed on their socio-demographic backgrounds consisting date of birth, age, gender and ethnic group. Investigation of patient's medical history on the diseases (with T2DM as a prerequisite) was conducted, which includes the presence of hypertension and dyslipidemia, duration of diseases and treatment obtained. Medical records of all patients were reviewed and extracted according to a standardized procedure.

\subsection{Anthropometric and Physiological Measurements}

Anthropometric measurements at the study visits included height, weight, WC, hip circumference and waist-to-hip ratio (WHR) were measured by trained personnel according to the standard procedures. Waist circumference was measured using a non-elastic tape made of fiberglass. Patients were asked to stand erect in a relaxed position with both feet together on a flat surface, while one layer of clothing was accepted. Waist girth was measured as the smallest horizontal girth between the costal margins and the iliac crests at minimal respira- 
tion, touching but not compressing the skin. For the statistical analysis, mean values of the two consecutive WC measurements were calculated. Hip circumference was measured to the nearest $0.5 \mathrm{~cm}$ by using the same nonelastic fiberglass tape over the greater trochanters (the widest portion of the hip) or the widest part of the gluteal region, respectively, with patients wearing light underwear. The WHR is an indicative of regional fat distribution, and it was determined using the formula as WC divided by hip circumference. Body mass index (BMI) was used as a measure of overall obesity. Body weight was measured to the nearest $0.1 \mathrm{~kg}$ by the SECA digital scale (THD-360, Tanita Health Equipment Ltd., Tokyo, Japan) with patients dressed in lightweight clothing (with heavy clothing removed and $0.5 \mathrm{~kg}$ deducted for remaining garments). Height was measured by a wall-mounted SECA microtoise tape (Model 206, Vogel and Halke GmbH \& Co., Hamburg, Germany) which was suspended upright against a smooth wall (with patients barefooted) in centimeters $(\mathrm{cm})$ (to the nearest $0.5 \mathrm{~cm}$ ) prior to conversion into meters $(\mathrm{m})$. Measurements were taken in duplicate and averaged. As an estimate of relative weight, BMI was computed as weight $(\mathrm{kg})$ divided by standing height squared $\left(\mathrm{m}^{2}\right)$. The WHO classification of BMI was used to classify the patients as underweight $\left(\mathrm{BMI}<18.5 \mathrm{~kg} / \mathrm{m}^{2}\right)$; normal (BMI 18.5 - $\left.24.9 \mathrm{~kg} / \mathrm{m}^{2}\right)$; overweight (BMI 25.0 - $29.9 \mathrm{~kg} / \mathrm{m}^{2}$ ); and obese (BMI > $30 \mathrm{~kg} / \mathrm{m}^{2}$ ) [23].

Blood pressure was measured at the sitting position using a calibrated digital Omron Automatic Blood Pressure Monitor (Model T8, Omron Healthcare Singapore Pte Ltd., Alexandra Technopark, Singapore) with an ap- propriate sized cuff on the left arm after taking rest for at least $10 \mathrm{~min}$. A cuff larger than the standard one was used when arm circumference exceeded $34 \mathrm{~cm}$. Mean values of the resting systolic and diastolic blood pressure were determined from two independent measurements at 5-min intervals. All measurements were taken by the same trained personnel to reduce error.

\subsection{Biochemical Parameters}

Information on the routine laboratory investigations involving glycemic control and plasma lipid profiles were retrieved from patients' medical records. The variables extracted from patients' records include glycated hemoglobin $\left(\mathrm{HbA}_{1 \mathrm{C}}\right)$, fasting plasma glucose (FPG), serum total cholesterol, high-density lipoprotein cholesterol (HDL-C), low-density lipoprotein cholesterol (LDL-C) and triglycerides. The last available values, i.e. the latest and must be of readings within the last three months were noted. Based on the Management of Type 2 Diabetes Mellitus Clinical Practice Guidelines criteria, $\mathrm{HbA}_{1 \mathrm{c}}$ and FPG of less than $6.5 \%$ and within $4.4-8.0$ $\mathrm{mmol} / \mathrm{L}$ respectively were considered as good glycemic control [24].

\subsection{Definition and Diagnosis of Metabolic Syndrome}

Table 1 manifests the summary of MetS classification in accordance to WHO, NCEP ATP III, IDF, and Harmonized definitions. The traditional risk factors of MetS were defined as follows: hypertension, dyslipidemia and $\mathrm{DM}$ as the use of antihypertensive, lipid lowering or

Table 1. WHO, NCEP ATP III, IDF and the Harmonized definitions of metabolic syndrome.

\begin{tabular}{|c|c|c|c|}
\hline WHO [1999] & NCEP ATP III [2001] & IDF [2006] & Harmonized [2009] \\
\hline $\begin{array}{l}\text { Diabetes (fasting plasma } \\
\text { glucose): } \geq 6.1 \mathrm{mmol} / \mathrm{L} \text { or } \\
\text { previously diagnosed T2DM }\end{array}$ & & $\begin{array}{l}\text { Central/ abdominal obesity: } \\
\mathrm{WC} \geq 90 \mathrm{~cm} \text { for Asian men, } \\
\text { and } \geq 80 \mathrm{~cm} \text { for Asian women }\end{array}$ & \\
\hline $\begin{array}{l}\text { Plus any two of the following } \\
\text { four factors: } \\
\text { Obesity: } \mathrm{BMI}>30 \mathrm{~kg} / \mathrm{m}^{2} \text {, or } \\
\text { WHR }>0.9 \text { in men } \\
\text { and }>0.85 \text { in women }\end{array}$ & $\begin{array}{l}\text { Three or more of the following } \\
\text { factors: } \\
\text { Central obesity: } \\
\text { WC }>102 \mathrm{~cm} \text { for men, } \\
\text { and }>88 \mathrm{~cm} \text { for women }\end{array}$ & $\begin{array}{l}\text { Plus any two or more of the } \\
\text { following four factors: }\end{array}$ & $\begin{array}{l}\text { Three or more of the following } \\
\text { factors: } \\
\text { Central/ abdominal obesity: } \\
\text { WC } \geq 90 \mathrm{~cm} \text { for Asian men, } \\
\text { and } \geq 80 \mathrm{~cm} \text { for Asian women }\end{array}$ \\
\hline $\begin{array}{l}\text { Raised triglycerides level: }>1.69 \\
\text { mmol/L; or specific treatment } \\
\text { for this lipid abnormality }\end{array}$ & $\begin{array}{l}\text { Raised triglycerides level: } \geq 1.69 \\
\text { mmol/L; or specific treatment } \\
\text { for this lipid abnormality }\end{array}$ & $\begin{array}{l}\text { Raised triglycerides level: } \geq 1.69 \\
\text { mmol/L; or specific treatment for } \\
\text { this lipid abnormality }\end{array}$ & $\begin{array}{l}\text { Raised triglycerides level: } \geq 1.69 \\
\text { mmol/L; or specific treatment for } \\
\text { this lipid abnormality }\end{array}$ \\
\hline $\begin{array}{l}\text { Reduced HDL-C: }<0.90 \mathrm{mmol} / \mathrm{L} \\
\text { in men, and }<1.0 \mathrm{mmol} / \mathrm{L} \text { in } \\
\text { women or on treatment for lipid } \\
\text { abnormality }\end{array}$ & $\begin{array}{l}\text { Reduced HDL-C: }<1.03 \mathrm{mmol} / \mathrm{L} \\
\text { in men, and }<1.29 \mathrm{mmol} / \mathrm{L} \text { in } \\
\text { women or on treatment for lipid } \\
\text { abnormality }\end{array}$ & $\begin{array}{l}\text { Reduced HDL-C: }<1.03 \mathrm{mmol} / \mathrm{L} \text { in } \\
\text { men, and }<1.29 \mathrm{mmol} / \mathrm{L} \text { in women } \\
\text { or on treatment for lipid } \\
\text { abnormality }\end{array}$ & $\begin{array}{l}\text { Reduced HDL-C: }<1.03 \mathrm{mmol} / \mathrm{L} \\
\text { in men, and }<1.29 \mathrm{mmol} / \mathrm{L} \text { in } \\
\text { women or on treatment for lipid } \\
\text { abnormality }\end{array}$ \\
\hline $\begin{array}{l}\text { Raised blood pressure: } \geq 140 / 90 \\
\text { mmHg or treatment of } \\
\text { previously diagnosed } \\
\text { hypertension }\end{array}$ & $\begin{array}{l}\text { Raised fasting plasma glucose: } \\
\geq 6.1 \mathrm{mmol} / \mathrm{L} \text { or previously } \\
\text { diagnosed T2DM }\end{array}$ & $\begin{array}{l}\text { Raised fasting plasma glucose: } \\
\geq 5.6 \mathrm{mmol} / \mathrm{L} \text { or previously } \\
\text { diagnosed T2DM }\end{array}$ & $\begin{array}{l}\text { Raised fasting plasma glucose: } \\
\geq 5.6 \mathrm{mmol} / \mathrm{L} \text { or previously } \\
\text { diagnosed T2DM }\end{array}$ \\
\hline
\end{tabular}

Abbreviations: BMI, body mass index; WHR, waist-to-hip ratio; WC, waist circumference; HDL-C, high-density lipoprotein cholesterol; T2DM, type 2 diabetes mellitus. 
anti-diabetic medication as reliably confirmed from the patient records. In our case, for the purpose of estimating the probable overall prevalence of MetS, the glycemic criterion was considered satisfied in all patients since they were diagnosed as having T2DM. The blood pressure criterion was considered satisfied if the patient was on any antihypertensive medications or if the blood pressure measured was above the target in patient who was not on any antihypertensive therapy. The same applies for dyslipidemia with respect to lipid lowering agents regardless of HDL-C and triglycerides levels, or if lipid profiles were above the target ranges. Generally, MetS was diagnosed when T2DM coexisted with $\geq 2$ other components of MetS based on the definitions described.

\subsection{Statistical Analysis}

All statistical analyses were performed by using IBM SPSS statistics Version 21.0 (SPSS Inc., Chicago, IL, USA). Descriptive statistics such as percentage (\%), mean, range and standard deviation (SD) were used to describe the data. A $p$ value of $<0.05$ was regarded as statistically significant. Kappa $(\kappa)$ statistics were used to estimate the agreement and concordance between the four definitions (WHO, NCEP ATP III, IDF and Harmonized). Kappa is always less than or equal to 1 , where the value of 1 implies perfect agreement and values less than 1 imply less than perfect agreement. More specifically, kappa indexes between 0.81 to 1.00 indicates a very good agreement, 0.61 to 0.80 represents good agreement, 0.41 to 0.60 means moderate agreement, 0.21 to 0.40 interpreted as fair agreement and a value of less than 0.20 reflects a weak agreement between the MetS definitions. Furthermore, we performed the sensitivity and specificity analyses to correctly detect those with and without the MetS based on all the definitions.

\section{RESULTS}

The details regarding socio-demographic, anthropometric, biochemical and clinical characteristics of patients are presented in Table 2. Generally, the mean age of the patients was $55.7 \pm 9.2$ years (ranged from 30 - 78 years), and their average duration of diabetes was approximately 10 years. Men and women were equally represented, and large proportions were Malays. The mean BMI, WC and WHR of the patients were $29.0 \pm 5.0 \mathrm{~kg} / \mathrm{m}^{2}, 96.3 \pm 11.2$ $\mathrm{cm}$ and $0.9 \pm 0.1$ respectively. The $\mathrm{HbA}_{\mathrm{lc}}$ levels of the patients ranged from $5.4 \%$ to $17.2 \%$ with a mean of $8.7 \pm$ $2.1 \%$, showing good-to-weak glycemic control. The high values of $\mathrm{HbA}_{1 \mathrm{c}}$ of patients were indicative of poor glycemic levels in the preceding three months, whilst only $14.1 \%$ demonstrated good glycaemic control. Similarly, the mean value for glucose measures of fasting plasma
Table 2. Socio-demographic, anthropometric, biochemical and clinical characteristics of type 2 diabetic patients $(n=313)$.

\begin{tabular}{|c|c|}
\hline Characteristic & $n(\%)$ or mean $\pm \mathrm{SD}$ \\
\hline \multicolumn{2}{|l|}{ Socio-demographic } \\
\hline Age (years) & $55.7 \pm 9.2$ \\
\hline $\begin{array}{l}\text { Gender } \\
\text { Male } \\
\text { Female }\end{array}$ & $\begin{array}{l}150(47.9) \\
163(52.1)\end{array}$ \\
\hline $\begin{array}{l}\text { Ethnicity } \\
\text { Malay } \\
\text { Chinese } \\
\text { Indian }\end{array}$ & $\begin{array}{l}147(47.0) \\
80(25.6) \\
86(27.5)\end{array}$ \\
\hline \multicolumn{2}{|l|}{ Anthropometric } \\
\hline Weight (kg) & $73.2 \pm 14.6$ \\
\hline Height $(\mathrm{cm})$ & $158.7 \pm 8.8$ \\
\hline Body mass index (BMI) $\left(\mathrm{kg} / \mathrm{m}^{2}\right)$ & $29.0 \pm 5.0$ \\
\hline $\begin{array}{l}\text { Underweight } \\
\text { Normal } \\
\text { Overweight } \\
\text { Obese }\end{array}$ & $\begin{array}{c}1(0.3) \\
64(20.5) \\
135(43.3) \\
112(35.9)\end{array}$ \\
\hline Waist circumference $(\mathrm{cm})$ & $96.3 \pm 11.2$ \\
\hline Hip circumference $(\mathrm{cm})$ & $102.5 \pm 10.5$ \\
\hline Waist-to-hip ratio (WHR) & $0.9 \pm 0.1$ \\
\hline \multicolumn{2}{|l|}{ Biochemical and Clinical } \\
\hline $\begin{array}{l}\text { Glycated hemoglobin }\left(\mathrm{HbA}_{1 \mathrm{c}}\right)(\%) \\
\leq 6.5 \% \text { (good) } \\
\quad>6.5 \% \text { (suboptimal) }\end{array}$ & $\begin{array}{l}8.7 \pm 2.1 \\
44(14.1) \\
269(85.9)\end{array}$ \\
\hline Fasting plasma glucose $(\mathrm{mmol} / \mathrm{L})$ & $8.8 \pm 3.6$ \\
\hline $\begin{array}{l}<4.4 \mathrm{mmol} / \mathrm{L} \\
4.4-8.0 \mathrm{mmol} / \mathrm{L} \\
>8.0 \mathrm{mmol} / \mathrm{L}\end{array}$ & $\begin{array}{c}5(1.6) \\
66(21.1) \\
242(77.3)\end{array}$ \\
\hline Total cholesterol (mmol/L) & $4.9 \pm 1.3$ \\
\hline $\begin{array}{l}\text { Low-density lipoprotein cholesterol } \\
(\text { LDL-C) }(\mathrm{mmol} / \mathrm{L})\end{array}$ & $2.9 \pm 1.1$ \\
\hline $\begin{array}{l}\text { High-density lipoprotein cholesterol } \\
\text { (HDL-C) }(\mathrm{mmol} / \mathrm{L})\end{array}$ & $1.2 \pm 0.3$ \\
\hline Triglycerides $(\mathrm{mmol} / \mathrm{L})$ & $1.8 \pm 1.2$ \\
\hline Systolic blood pressure (SBP) (mmHg) & $137.9 \pm 18.9$ \\
\hline Diastolic blood pressure (DBP) (mmHg) & $80.7 \pm 11.8$ \\
\hline
\end{tabular}

Abbreviation: SD, standard deviation.

glucose was $8.8 \pm 3.6 \mathrm{mmol} / \mathrm{L}$. In addition, the mean total cholesterol, LDL-C, HDL-C and triglycerides levels were $4.9 \pm 1.3 \mathrm{mmol} / \mathrm{L}, 2.9 \pm 1.1 \mathrm{mmol} / \mathrm{L}, 1.2 \pm 0.3$ $\mathrm{mmol} / \mathrm{L}$ and $1.8 \pm 1.2 \mathrm{mmol} / \mathrm{L}$ respectively. The most commonly prescribed anti-diabetic therapy was oral anti-diabetic agents $(60.5 \%)$, followed by combination of oral agents and insulin (31.5\%) and intensive insulin monotherapy $(8.0 \%)$. Majority of the patients were receiving lipid lowering agents $(89.1 \%)$ and antihypertensive medications $(80.2 \%)$ with a mean SBP/DBP of $137.9 / 80.7 \mathrm{mmHg}$.

An alarmingly high prevalence of MetS was found among the type 2 diabetic patients regardless of the definitions used. The overall prevalence of MetS ( $\geq 3$ of 5 components) (n, 95\% CI) was 95.8\% (297, 93.6 - 98.1), 
$96.1 \%(298,94.0-98.3), 84.8 \%(263,80.8-88.9)$, and $97.7 \%(303,96.1$ - 99.4) under the WHO, NCEP ATP III, IDF, and Harmonized definitions, respectively. The latest Harmonized definition outperformed the other definitions in diagnosing MetS among the patients. The prevalence of various combinations of MetS components defined by WHO, NCEP ATP III, IDF and Harmonized criteria are denoted in Figure 1. Most patients met criteria for MetS, and the proportion of patients without MetS was relatively small. As much as over $95 \%$ of the type 2 diabetic patients were classified as fulfilling three, four or five MetS components by all the WHO, NCEP ATP III, IDF and Harmonized criteria. It is worth noting that only a few patients $(<5 \%)$ had one MetS component accompanying T2DM. The prevalence of individual components of the MetS in the patients is exhibited in Table 3. From the individual MetS components point of view, the low HDL-C criterion was most frequently documented in type 2 diabetic patients as defined according to NCEP ATP III (95.5\%), WHO (92.3\%), IDF (95.5\%) and Harmonized (95.5\%) definitions. This was followed by hypertriglyceridemia $(92.9 \%$ adopted as per all the MetS definitions), hypertension (NCEP ATP III, 90.4\%; WHO, 87.2\%; IDF, 90.4\%; and Harmonized criteria, $90.4 \%$ ), and obesity. Among the patients classified as having obesity, more than half $(59.3 \%, 59.3 \%$ and $51.3 \%)$ fulfilled the Harmonized, IDF and NCEP ATP III definitions for central obesity (WC criterion), whereas $86.5 \%$ and $35.9 \%$ met WHO definition for WHR and BMI, re- spectively.

The concordance as well as sensitivity and specificity of the MetS for the referenced definitions were determined as depicted in Table 4 . The $\kappa$ statistics confirmed a significant agreement between the definitions. The WHO against NCEP ATP III criteria had the best agreement with the highest kappa index $(\kappa=0.875, p<0.001)$ and sensitivity $(99.66 \%)$. This was followed by Harmonized-NCEP ATP III $(\kappa=0.729, p<0.001$; sensitivity $=$ $98.35 \%$; specificity $=100 \%)$ and Harmonized-WHO $(\kappa$ $=0.691, p<0.001$; sensitivity $=98.02 \%$; specificity $=$ $100 \%$, all indicating good agreements. The IDF data added nothing to the agreements regarding all the three Harmonized, NCEP ATP III and WHO definitions as can be clearly seen from the unsatisfactory kappa indexes, thus, were not further considered for sensitivity and specificity of MetS diagnosis.

\section{DISCUSSIONS}

This is the first report as per the authors' knowledge, which quantifies the prevalence of MetS among type 2 diabetic patients in Malaysia and determines the agreement of the various MetS definitions. Outcomes of our study are congruent with, and in fact considerably higher than the previous studies that clarified high prevalence of MetS among diabetic participants ranging from 50\% to $92.3 \%[10,12,14,25-31]$. The alarmingly high prevalence of MetS among our patients is hardly surprising. Besides $\mathrm{DM}$ itself as a predominant CVD risk factor, another

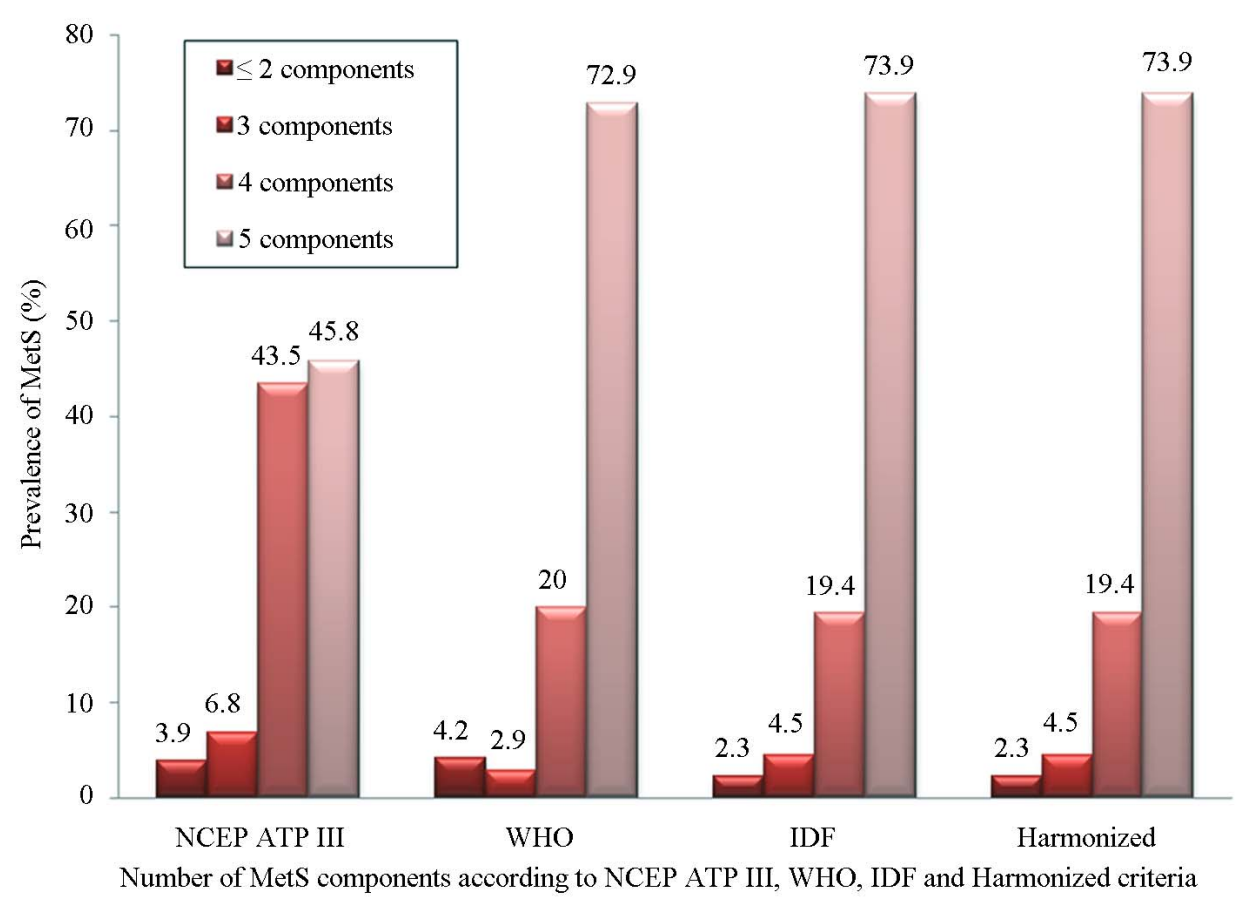

Figure 1. Proportion of patients with 1 to 5 components of MetS in accordance with NCEP ATP III, WHO, IDF and Harmonized criteria, respectively $(n=313)$. 
Table 3. Prevalence of individual MetS components according to WHO, NCEP ATP III, IDF and Harmonized criteria $(n=313)$.

\begin{tabular}{lcccc}
\hline Criterion & WHO & NCEP ATP III & IDF & Harmonized \\
\hline Obesity & $112(35.9)$ & - & - & - \\
$\quad$ BMI (\%) & - & $160(51.3)$ & $267(85.6)$ & $267(85.6)$ \\
WC (\%) & $270(86.5)$ & - & - & - \\
WHR (\%) & $273(87.2)$ & $283(90.4)$ & $283(90.4)$ & $283(90.4)$ \\
Hypertension (\%) & $287(92.3)$ & $297(95.5)$ & $297(95.5)$ & $297(95.5)$ \\
Low HDL-C (\%) & $290(92.9)$ & $290(92.9)$ & $290(92.9)$ & $290(92.9)$ \\
Elevated triglycerides (\%) & & & \\
\hline
\end{tabular}

Data presented as $n(\%)$; Abbreviations: BMI, body mass index; WC, waist circumference; WHR, waist-to-hip ratio; HDL-C, high-density lipoprotein cholesterol.

Table 4. The agreement and disparity in diagnosis of MetS using the NCEP ATP III, WHO, IDF and Harmonized definitions.

\begin{tabular}{|c|c|c|c|c|c|c|c|}
\hline Definitions & Non-MetS & MetS & Sensitivity (\%) & Specificity (\%) & Kappa index & $p$ value & Agreement \\
\hline WHO against NCEP ATP III & & & 99.66 & 84.62 & 0.875 & $<0.001$ & Very good \\
\hline Non-MetS & 11 & 1 & & & & & \\
\hline MetS & 2 & 296 & & & & & \\
\hline Harmonized against NCEP ATP III & & & 98.35 & 100 & 0.729 & $<0.001$ & Good \\
\hline Non-MetS & 7 & 5 & & & & & \\
\hline MetS & 0 & 298 & & & & & \\
\hline Harmonized against WHO & & & 98.02 & 100 & 0.691 & $<0.001$ & Good \\
\hline Non-MetS & 7 & 6 & & & & & \\
\hline MetS & 0 & 297 & & & & & \\
\hline Harmonized against IDF & & & 86.80 & 100 & 0.229 & $<0.001$ & Fair \\
\hline Non-MetS & 7 & 40 & & & & & \\
\hline MetS & 0 & 263 & & & & & \\
\hline IDF against NCEP ATP III & & & 98.10 & 14.89 & 0.187 & $<0.001$ & Weak \\
\hline Non-MetS & 7 & 5 & & & & & \\
\hline MetS & 40 & 258 & & & & & \\
\hline IDF against WHO & & & 97.72 & 14.89 & 0.179 & $<0.001$ & Weak \\
\hline Non-MetS & 7 & 6 & & & & & \\
\hline MetS & 40 & 257 & & & & & \\
\hline
\end{tabular}

factor that explains the high prevalence of MetS is the age, where almost all of the patients who participated in the study aged over 40 years old (93.9\%). As commonly known, MetS is more prevalent as age increases. Moreover, the study subjects were tertiary hospital-based patients with DM of a relatively long duration; it is likely that these patients have more diabetic complications and a more difficult metabolic control than expected in a group of patients with T2DM followed up by general practitioners in primary care settings.

There are important differences between the WHO, NCEP ATP III, IDF and Harmonized definitions of the MetS that may explain the dissimilar magnitudes of MetS prevalence. Firstly, a much lower cut-off value for WC in the IDF and Harmonized definitions compared to NCEP ATP III criteria leads to the inclusion of patients with a relatively lower level of this risk factor. Secondly, the compulsory inclusion of the WC criterion in the IDF definition employed $(\geq 90 \mathrm{~cm}$ in males and $\geq 80 \mathrm{~cm}$ in females) results in a relatively lower prevalence of the other potentially stronger MetS risk factors. This also implies that the IDF definition erroneously overlooked patients that actually had MetS. On the contrary, in the Harmonized criteria, those non-obese patients having other components of MetS can be categorized as having MetS as long as they met the minimum numbers of components required, resulting in more patients being diagnosed as having MetS. In terms of concordance, the disagreement of IDF with other criteria can be explained by the reason that central obesity was made mandatory in the IDF definition.

There was an excellent agreement observed between 
WHO and NCEP ATP III definitions of MetS. The high degree of overlap is not surprising considering four out of the five criteria of the two definitions are identical or nearly identical, despite several noteworthy discrepancies. In spite of the WHO definition requires the presence of DM or hyperglycemia, individuals with T2DM are automatically considered to have hyperglycemia even if their concentration of fasting plasma glucose may be lower than the cut-off point, resulting WHO definition makes DM one of the five equally weighted criteria which is similar to NCEP ATP III definition in classifying our type 2 diabetic patients having or not having MetS. Likewise, NCEP ATP III and Harmonized definitions which share common MetS components yielded good agreements between Harmonized-NCEP ATP III and Harmonized-WHO definitions, although they were only slightly weaker than the agreement between WHO and NCEP ATP III definition. The thresholds for concentrations of triglycerides, HDL-C, and fasting plasma glucose and for systolic and diastolic blood pressure vary slightly between these definitions, with the obvious difference being the obesity cut-off points between the definitions. However, the MetS definitions explicitly allow for the treatment of hypertriglyceridemia, low concentration of HDL-C, hypertension, and DM to be counted, resulting in similar and compatible MetS prevalence and agreements between the definitions for MetS diagnosis.

\section{CONCLUSIONS}

Metabolic syndrome was found to be relatively common in type 2 diabetic patients as demonstrated by the alarmingly high prevalence documented using WHO, NCEP ATP III, IDF and the new Harmonized criteria. The new Harmonized criteria established the highest prevalence of MetS, followed by NCEP ATP III, IDF, and WHO definitions. There was a strong concordance between the WHO and NCEP ATP III criteria. The WHO against NCEP ATP III criteria evinced the highest sensitivity whereas Harmonized criteria against all the other three definitions showed the highest specificity in identifying MetS.

The identification of MetS among type 2 diabetic patients is of great importance, since this population carries a cluster of cardiovascular risk factors and therefore, should be urged to show maximum compliance to their therapeutic regimen and healthy lifestyle. Nevertheless, the co-existence of multiple disorders makes it difficult to identify the therapeutic priorities. Obesity, DM, hypertension and dyslipidemia may require multi-drug treatment, whereas a comprehensive approach and educational programs may be beneficial to all components of MetS. Lifestyle interventions are possible and effective. It is time for health care providers to reconsider resource allocation for cost-effective behavioral strate- gies.

\section{ACKNOWLEDGEMENTS}

This study was supported by the Research University Grant Scheme and Department of Medicine, Universiti Putra Malaysia (UPM) (RUGS 9199609) (Principal Investigator: Dr. Ng Ooi Chuan).

\section{REFERENCES}

[1] American Heart Association (2009) Heart disease and stroke statistics: 2009 update. American Heart Association, Dallas.

[2] Alberti, K.G., Zimmet, P. and Shaw, J. (2005) The metabolic syndrome: A new worldwide definition. Lancet, $\mathbf{3 6 6}$, 1059-1062.

http://dx.doi.org/10.1016/S0140-6736(05)66835-3

[3] NCEP Expert Panel (2001) Executive summary of the third report of the National Cholesterol Education Program (NCEP) Expert Panel on detection, evaluation, and treatment of high blood cholesterol in adults (Adult Treatment Panel III). JAMA, 285, 2486-2497. http://dx.doi.org/10.1001/jama.285.19.2486

[4] IDF (2006) The IDF consensus worldwide definition of the metabolic syndrome.

http://www.idf.org/metabolic syndrome

[5] WHO (1999) Definitions, diagnosis and classification of diabetes mellitus and its complication. Report of a WHO consultation. Department of Non-Communicate Disease Surveillance, Geneva.

[6] American Heart Association (2012) Heart disease and stroke statistics-2012 update: A report from the American Heart Association. Circulation, 125, e2-e220.

[7] Alberti, K.G., Eckel, R.H., Grundy, S.M., Zimmet, P.Z., Cleeman, J.I., Donato, K.A., et al. (2009) Harmonizing the metabolic syndrome: A joint interim statement of the International Diabetes Federation Task Force on Epidemiology and Prevention; National Heart, Lung, and Blood Institute; American Heart Association; World Heart Federation; International Atherosclerosis Society; and International Association for the Study of Obesity. Circulation, 120, 1640-1645. http://dx.doi.org/10.1161/CIRCULATIONAHA.109.1926 $\underline{44}$

[8] Afsana, F., Latif, Z.A., Khan, S.J. and Talukder, S.K. (2010) Metabolic syndrome and cardiovascular risk in diabetic subjects. CVD Prevention and Control, 5, 59-62. http://dx.doi.org/10.1016/j.cvdpc.2010.05.001

[9] Kendall, D.M. and Harmel, A.P. (2002) The metabolic syndrome, type 2 diabetes, and cardiovascular disease: Understanding the role of insulin resistance. American Journal of Management Care, 8, S635-S653.

[10] Bonora, E., Targher, G., Formentini, G., Calcaterra, F., Lombardi, S., Marini, F., et al. (2003) The metabolic syndrome is an independent predictor of cardiovascular disease in type 2 diabetic subjects. Prospective data from the Verona Diabetes Complications Study. Diabetic Medicine, 21, 52-58. 
http://dx.doi.org/10.1046/j.1464-5491.2003.01068.x

[11] Nakanishi, N., Takatorige, T., Fukuda, H., Shirai, K., Li, W., Okamotoa, M., et al. (2004) Components of the metabolic syndrome as predictors of cardiovascular disease and type 2 diabetes in middle-aged Japanese men. Diabetes Research and Clinical Practice, 64, 59-70. http://dx.doi.org/10.1016/j.diabres.2003.10.001

[12] Guzder, R.N., Gatling, W., Mullee, M.A. and Byrne, C.D. (2006) Impact of metabolic syndrome criteria on cardiovascular disease risk in people with diagnosed type 2 diabetes. Diabetologia, 49, 49-55. http://dx.doi.org/10.1007/s00125-005-0063-9

[13] Kim, W.Y., Kim, J.E., Choi, Y.J. and Huh, K.B. (2008) Nutritional risk and metabolic syndrome in Korean type 2 diabetes mellitus. Asia Pacific Journal of Clinical Nutrition, 17, 47-51.

[14] AlSaraj, F., McDermott, J.H., Cawood, T., McAteer, S., Ali, M., Tormey, W., et al. (2009) Prevalence of the metabolic syndrome in patients with diabetes mellitus. Ireland Journal of Medical Sciences, 178, 309-313. http://dx.doi.org/10.1007/s11845-009-0302-z

[15] Thorn, L.M., Forsblom, C., Fagerudd, J., Thomas, M.C., Pettersson-Fernholm, K., Saraheimo, M., et al. (2005) Metabolic syndrome in type 1. Diabetes, 28, 2019-2014.

[16] Eckel, R.H., Grundy, S.M. and Zimmet, P.Z. (2005) The metabolic syndrome. The Lancet, 365, 1415-1428. http://dx.doi.org/10.1016/S0140-6736(05)66378-7

[17] Zimmet, P., Magliano, D., Matsuzawa, Y., Alberti, G. and Shaw, J. (2005) The metabolic syndrome: A global public health problem and a new definition. Journal of Atherosclerosis and thrombosis, 12, 295-300. http://dx.doi.org/10.5551/jat.12.295

[18] Ford, E.S. (2005) Prevalence of the metabolic syndrome defined by the International Diabetes Federation among adults in the U.S. Diabetes Care, 28, 2745-2749.

[19] Lien, L.F. and Guyton JR. (2008) Metabolic syndrome. Dermatologic Therapy, 21, 362-375. http://dx.doi.org/10.1111/j.1529-8019.2008.00218.x

[20] Grundy, S.M., Cleeman, J.I., Daniels, S.R., Donato, K.A., Eckel, R.H., Franklin, B.A., et al. (2005) Diagnosis and management of the metabolic syndrome: An American Heart Association/National Heart, Lung and Blood Institute scientific statement. Circulation, 112, 2735-2752. http://dx.doi.org/10.1161/CIRCULATIONAHA.105.1694 $\underline{04}$

[21] Huang, P.L. (2009) A comprehensive definition for metabolic syndrome. Disease Models \& Mechanisms, 2, 231-
237. http://dx.doi.org/10.1242/dmm.001180

[22] MOH Malaysia (2011) Malaysian guideline for good clinical practice. 3rd Edition, MOH Malaysia, Putrajaya.

[23] WHO (1995) Physical Status: The use and interpretation of anthropometry. Report of a WHO Expert Committee. WHO Technical Report Series 854. WHO, Geneva.

[24] MOH Malaysia (2009) Clinical practice guidelines: Management of Type II Diabetes Mellitus. 4th Edition, $\mathrm{MOH}$ Malaysia, Putrajaya.

[25] Orna, J.A.G., Arnal, L.M.L., Herguedas, E.M., Julián, B.B. and Córdobaa, D.P.P. (2004) Metabolic syndrome as a cardiovascular risk factor in patients with type 2 diabetes. Revista Española de Cardiología, 57, 507-513.

[26] Lu, W., Resnick, H.E., Jablonski, K.A., Jones, K.L., Jain, A.K., Howard, W.J., et al. (2003) Non-HDL cholesterol as a predictor of cardiovascular disease in type 2 diabetes: The strong heart study. Diabetes Care, 26, 16-23. http://dx.doi.org/10.2337/diacare.26.1.16

[27] Isomaa, B., Henricsson, M., Almgren, P., Tuomi, T., Taskinen, M.R. and Groop, L. (2001b) The metabolic syndrome influences the risk of chronic complications in patients with type II diabetes. Diabetologia, 44, 1148-1154. http://dx.doi.org/10.1007/s001250100615

[28] Protopsaltis, I., Nikolopoulos, G., Dimoua, E., Brestas, P., Kokkoris, S., Korantzopoulos, P., et al. (2007) Metabolic syndrome and its components as predictors of all-cause mortality and coronary heart disease in type 2 diabetic patients. Atherosclerosis, 195, 189-194. http://dx.doi.org/10.1016/j.atherosclerosis.2006.09.019

[29] Monami, M., Masotti, N.M.G. and Mannucci, E. (2007) IDF and ATP-III definitions of metabolic syndrome in the prediction of all-cause mortality in type 2 diabetic patients. Diabetes, Obesity and Metabolism, 9, 350-353. http://dx.doi.org/10.1111/j.1463-1326.2006.00615.x

[30] Hanefeld, M., Koehler, C., Gallo, S., Benke, I. and Ott, P. (2007) Impact of the individual components of the metabolic syndrome and their different combinations on the prevalence of atherosclerotic vascular disease in type 2 diabetes: The Diabetes in Germany (DIG) study. Cardiovascular Diabetology, 6, 13. http://dx.doi.org/10.1186/1475-2840-6-13

[31] Bruno, G., Merletti, F., Biggeri, A., Bargero, G., Ferrero, S., Runzo, C., et al. (2004) Metabolic syndrome as a predictor of all-cause and cardiovascular mortality in type 2 diabetes: The Casale Monferrato study. Diabetes Care, 27, 2689-2694. http://dx.doi.org/10.2337/diacare.27.11.2689 